\title{
Fisherian and Wrightian perspectives in evolutionary genetics and model-mediated imposition of theoretical assumptions
}

\author{
Rasmus Grønfeldt Winther* \\ Instituto de Investigaciones Filosóficas, Circuito Mario de la Cueva s/n, Universidad Nacional Autónoma de México, Coyoacán 04510, Mexico City, México
}

Received 26 February 2005; received in revised form 13 September 2005; accepted 14 September 2005

Available online 2 November 2005

\begin{abstract}
I investigate how theoretical assumptions, pertinent to different perspectives and operative during the modeling process, are central in determining how nature is actually taken to be. I explore two different models by Michael Turelli and Steve Frank of the evolution of parasite-mediated cytoplasmic incompatility, guided, respectively, by Fisherian and Wrightian perspectives. Since the two models can be shown to be commensurable both with respect to mathematics and data, I argue that the differences between them in the (1) mathematical presentation of the models, (2) explanations, and (3) objectified ontologies stem neither from differences in mathematical method nor the employed data, but from differences in the theoretical assumptions, especially regarding ontology, already present in the respective perspectives. I use my "set up, mathematically manipulate, explain, and objectify" (SMEO) account of the modeling process to track the model-mediated imposition of theoretical assumptions. I conclude with a discussion of the general implications of my analysis of these models for the controversy between Fisherian and Wrightian perspectives.
\end{abstract}

(C) 2005 Elsevier Ltd. All rights reserved.

Keywords: Fisher; Wright; Cytoplasmic incompatibility; Perspectives; Theories; Models; Assumptions; Evolutionary genetics; Parasite evolution

\section{Context}

The controversy between Fisherian and Wrightian approaches to evolution has been, and continues to be, an arduous one (historical and philosophical analyses include Provine, 1986; Lloyd, 1994, 2000; Gayon, 1998; Morrison, 2000; Skipper, 2002). A recent series of exchanges between Jerry Coyne, Nick Barton, and Michael Turelli, and Michael Wade and Charles Goodnight, in the pages of Evolution, highlights a number of the relevant issues. In this article, I show that, to an extent at least, these two groups of authors share an important set of presuppositions regarding the theory-data relation-namely, that data are independent of theory, and that theory can be straightforwardly evaluated by theory-independent data. I argue that there is another way to look at the theory-data relation that focuses on the model-mediated imposition of theory onto data and, ultimately, onto nature (since nature is seen as causing data). I do not defend my analysis as a

\footnotetext{
${ }^{*}$ Tel.: + 525556227444 ; fax: + 525556227376 .

E-mail address: rgwinther@gmail.com.
}

complete picture of the theory-data relation. However, I do claim that it is necessary to consider an alternative to the usual way of thinking about this relation.

My argument consists of three parts. In the remainder of this section, I motivate the existence of model-mediated imposition of theoretical assumptions onto data and nature (or, to abbreviate, theoretical imposition) in the recent controversy between Fisherian and Wrightian approaches. In Section 2, I provide a general account of the modeling process, which I call the SMEO ("set up, mathematically manipulate, explain, and objectify") account, that allows for the tracking of theoretical imposition. In Section 3, I apply the SMEO account to the particular case of two models, Turelli's Fisherian model and Steve Frank's Wrightian model, ${ }^{1}$ of the evolution of parasite-induced

\footnotetext{
${ }^{1}$ It is important to note that I am considering the controversy using the contemporary articulations of the two perspectives. Coyne et al. observe: "There is thus a clear distinction between the Fisherian and Wrightian views of evolution: the former requires only that populations be larger than the reciprocal of the selective coefficient acting on a genotype, and the latter requires sub-divided populations, particular forms of epistasis, genetic drift that counteracts selection, and differential migration between
} 
cytoplasmic incompatibility (CI). As I will argue later in this section, this case of modeling is a particularly useful example of theoretical imposition. In my conclusion, I show how my examination of these two particular models of CI evolution informs the general controversy between Fisherian and Wrightian perspectives.

The two groups of authors accept that theory and its models explain, and are evaluated by, data that are both independent of, and fairly robust to, theoretical context. On the one hand, Coyne et al. "favor the view that adaptations are usually produced by Fisherian mass selection, a process that is not only more parsimonious than the SBT [Wright's Shifting Balance Theory], but has also been shown to occur widely (Endler, 1986)." (Coyne et al., 1997, p. 665). Both parsimony ${ }^{2}$ and explanatory power are the two main reasons for why they "believe that most adaptations in nature can be explained by natural selection acting on intrapopulation variation." (Coyne et al., 2000, p. 306; in his analysis, Skipper (2002) focuses on parsimony). Underlying their argument is the view that Fisherian mass selection is empirically adequate and empirically sufficient to explain theory-independent data.

On the other hand, Wade and Goodnight claim that each theory is incomplete. For them, the task at hand is to characterize the respective domains of applicability (see Skipper, 2002). They write:

We conclude that both [Fisher's and Wright's] theories, at least in their idealized original versions, have difficulty incorporating important features of natural populations... because of the assumptions that differ between them. We argue that this limits the application of each theory to different domains. (Goodnight and Wade, 2000, p. 317)

That is, the conditions of application depend on the relevance, precision, and realism of the theoretical assumptions intrinsic to each perspective. For example, Wade and Goodnight argue that in the domain of speciation and evolution in metapopulations, Wright's theory is more

\section{(footnote continued)}

populations based on their genetic constitution." (Coyne et al., 1997, p. 644). Wade and Goodnight write: "Although the mathematical details of these theories are largely in agreement, the conceptual emphases of Wright and Fisher were so different... that where and how to apply the theories to the natural world has been and continues to be a source of controversy." (Wade and Goodnight, 1998, p. 1537, emphasis mine). I am not making exegetical claims about what Fisher or Wright themselves actually believed, since there are separate interpretative questions about this. For example, Steve Frank (pers. comm.; Frank and Slatkin, 1992) defends an interpretation of Fisher's work in which, roughly, Fisher was not a Fisherian, sensu contemporary debates-he defended the importance of genetic epistasis, for example. These important interpretative questions about Fisher's and Wright's work are beyond the scope of my article.

${ }^{2}$ As one reviewer pointed out, the argument here seems to be that the cooccurrence of the multiple evolutionary forces implied by the SBT is very improbable. Due to considerations of parsimony, the Fisherian perspective, then, is considered the more likely theory by these authors (on the complex connections among parsimony, probability, and the likelihood of a hypothesis, see Sober, 2003). successful empirically and theoretically. Note that they discuss the existence and nature of these domains independently of any theory. Their concern with success of application is a concern with the empirical adequacy of models.

Coyne et al. as well as Wade and Goodnight (to a lesser extent $t^{3}$ ), thus agree on three theses: (1T) empirical adequacy is the main evaluation criterion in the process of theory choice, (2T) the data are epistemically and methodologically independent of theory and modeling, and (3T) theory choice (which here employs empirical adequacy) occurs through evaluating the degree of matching or fitting of information across two roughly independent domains: theory/model and data/nature. In contrast to (1T), I want to point to the existence of considerations besides empirical adequacy as operative in the process of theory choice, including commitment to a perspective due to its perceived simplicity or unifying power, or because it is part of a research tradition to which one belongs. In contrast to (2T) and (3T), I want to highlight the process of imposing theoretical assumptions onto data and, ultimately, nature (see Kuhn, 1970; Levins and Lewontin, 1985). An extreme commitment to theoretical imposition, which I do not support, would vigorously deny all three theses stated. What I will defend, for the purpose of discussing the important role of theory, models, and modeling in imposing assumptions about ontology, is a constrained denial of the three theses. Ultimately, such a constrained denial should be compared and intertwined with the assertion of these theses, as well as with other views regarding the theory-data relation, in order to develop as complete an understanding of this relation as possible.

Before turning to my analysis, I want to discuss two sets of phenomena which both groups of authors explicitly address: family level selection in chickens and CI evolution. The first example allows us to clearly see theoretical imposition on data. Although the second case is the focus of this article, I mention it here because it is important to clarify the reasons for its utility for observing and understanding theoretical imposition.

The fact that there are two differing interpretations of family level selection in chickens provides clear evidence for theoretical imposition. Briefly, Muir (1996) and Craig and Muir (1996) increased egg-laying rates in domestic chickens by selecting cages of full-sib sisters with high egglaying rates, instead of selecting individuals with high egglaying rates (see also the further analysis in Muir, 2005). Their selection protocol was extremely successful. Wade and Goodnight interpret this as indicating the "efficacy of intergroup selection." (Wade and Goodnight, 1998, p. 1538). Individual selection, which had been practiced

\footnotetext{
${ }^{3}$ Wade (pers. comm.) also accepts the alternative interpretation of the theory-data relation I defend in that he endorses a position, adopted by Neyman et al. (1956), that articulates the complex interaction among theory, experiment, statistics, and data.
} 
since 1925, was no longer effective; group selection was much more successful than individual selection in changing the trait mean (Goodnight and Wade, 2000, p. 321). They furthermore argue that this is family, kin, and group selection: "there are many circumstances in which kin selection can be considered a form of group selection (e.g. Bourke and Franks, 1995, ch. 2; Crozier and Pamilo, 1996, pp. 25-27; Frank, 1998; Michod, 1999)." (Goodnight and Wade, 2000, p. 321).

On the other hand, Coyne et al, argue that this is at best a case of family level selection in which individual selection control lines were not established, thereby making it impossible to formulate any claims about the relative force of individual vs. family level selection. Furthermore, and more importantly, the appropriate level of selection remains individual selection since the selection protocol employed "is a well known method of animal breeding that is used to alter traits of low heritability because it effectively reduces environmental variance and allows selection for traits that cannot be scored in all individuals (Falconer and Mackay, 1996, ch. 13)." This could also be a case in which selection "operate[s] more rapidly [because] inbreeding increases the frequency of the advantageous phenotype (e.g. Caballero et al., 1991)." (Coyne et al., 2000 , p. 309). For these authors, the process of selection is occurring at the individual level.

Another way to state the difference, as one reviewer of my article noted, is that housing families in the same cage implies two things: (1) the chickens were forced to interact, and (2) the cage represents a kind of summary statistic of the family. Wade and Goodnight highlight the first consequence, whereas Coyne et al. emphasize the second implication.

Thus, Wade and Goodnight insist that this is a case of group-level selection, whereas Coyne, Barton, and Turelli construe the experiment as a standard textbook protocol of individual-level selection. From this brief exposition of the two interpretations of Craig and Muir's experiments, it should be clear that the same set of data (and process of selection) can be interpreted from, and actively made compatible with, either perspective. The data are not inherently neutral vis-à-vis theory [contra thesis (2T)], nor is theory inert in terms of establishing the very relation between theory and data [contra thesis (3T)].

I will now turn to two reasons for choosing CI evolution as a paradigmatic case of theoretical imposition. First, scientists from both perspectives have provided impressive models of this single process. Turelli, one of the co-authors of the Fisherian articles, provides a model that, because of its primary assumptions of panmixia and general absence of kin selection, can be readily interpreted as Fisherian; Frank, who elsewhere defends population structure and kin selection as common and effective in the evolutionary process (e.g. Frank, 1994, 1998), presents a model that is committed to these processes and can, therefore, be considered Wrightian. Thus, CI evolution provides a kind of "controlled conceptual experiment" of the nature of modeling since process studied and mathematical method used are held constant - there is basically no variance in them across the two models. Differences in outcome of this case - the distinct modeling conclusions - stem largely from variance in theoretical assumptions across the two perspectives.

The second reason for the utility of this case is that biologists from both perspectives admit that due to certain peculiar features of CI evolution, to be explored below, it can be adequately analysed from a Wrightian perspective. For example, in their critique of Wright's Shifting Balance Theory, Coyne et al. note that:

Two of the cases (cytoplasmic incompatibility in Drosophila and coiling in snails) also appear to show that populations evolved past unstable equilibria to new peaks (phase II). Only one of these cases, however (cytoplasmic incompatibility), shows convincing evidence for phase III [interdemic selection], and none show that the entire process has led to higher population fitness. (Coyne et al., 1997, p. 661)

Thus, despite being critical of Wright's theory, they admit that parasite-induced CI requires interdemic selection and population structure; they do not, however, mention kin selection. It is therefore all the more surprising that in his close analysis of the evolutionary dynamics of CI, Turelli develops a strongly Fisherian model (Turelli, 1994). Again, this provides evidence for theoretical imposition: a particular set of phenomena, first admitted to be adequately interpreted as falling under one theory, is subsequently formulated, in detail, as subsumable under an alternative theory. In short, the case of CI evolution is useful for showing theoretical imposition because it is a single process studied from two vantage points, and because both perspectives agree that it fits the Wrightian perspective better, yet the Fisherian perspective reinterprets it as a Fisherian process. Let us now turn to the models.

\section{The SMEO account of modeling}

I want to present a simplified and linear account of the modeling process, which I call the SMEO account. ${ }^{4}$ Levins and Lewontin are important, among other reasons, for clearly diagnosing a variety of limitations with modelbuilding, including intrinsic trade-offs in modeling (e.g. Levins, 1966, 1968), as well as problems surrounding the dynamic sufficiency and empirical sufficiency of models (e.g. Lewontin, 1974). In their 1985 book, they issue a note

\footnotetext{
${ }^{4}$ I do not wish to state that my account is objective or that it is the only way of representing how theoretical imposition occurs in mathematical modeling. There is, admittedly, a problem of self-reflexivity here. If I claim that modeling always involves theoretical imposition, must I not analyze my own account (model) for this? I believe that because of the utility of my account in tracking biases and pre-suppositions in mathematical modeling, it is reasonable to side-step this question for now, while fully admitting that any number of imposed biases and theoretical assumptions undoubtedly exist in my account.
} 
of warning regarding the process of abstraction (abstraction is integral to the process of model-building):

We can hardly have a serious discussion of a science without abstraction. What makes science materialist is that the process of abstraction is explicit and recognized as historically contingent within the science. Abstraction becomes destructive when the abstract is reified and when the historical process of abstraction is forgotten, so that the abstract descriptions are taken for descriptions of the actual objects. ... The problem for science is to understand the proper domain of explanation of each abstraction rather than become its prisoner. (Levins and Lewontin, 1985, pp. 149-150)

I take this warning seriously. My SMEO account allows us to track "when the abstract is reified" in order to not forget "the historical process of abstraction." My account has been inspired by a variety of suggestions concerning the modeling process by a number of biologists and philosophers, including, besides Levins and Lewontin, Kuhn (1970), Wimsatt (1974, 1980, 1987, 1994), van Fraassen (1980, 1989), Cartwright (1983, 1999), Oyama (1985), Lloyd (1988), Griesemer (1990), Smith (1996), Morgan and Morrison (1999), and Morrison (2000). I divide the modeling process into four stages and will now explore each in turn.

In the first step, the theory provides the frame for setting up the model. Theoretical assumptions of various sorts pick out what are interpreted as the important material structures and processes of the system under study. These assumptions concern views both about basic ontology, including basic structures and basic causes, and about legitimate ways of abstracting; they are themselves the product of previous theoretical and empirical activity. The setup of the model involves assigning parameters and variables to the key properties of the chosen structures and processes, as well as capturing the relations between these properties in the initial equations of the model. In short, the theoretical assumptions determine the fundamental content and form of the model. (Step 1-S: here the model is set up.)

In the second step, these initial equations, which are seen as basic to the dynamics of the system, are subsequently manipulated. Sometimes, surprising results (e.g. unexpected final equations or equilibrium conditions) are derived. In this step, many techniques and assumptions pertinent to mathematics are instantiated and employed. Furthermore, the kinds of approximations and idealizations made, and heuristics used, during mathematical manipulation are also justified by the operative theory. In the activity of modeling, mathematical content, rather than material content, is manipulated. The latter sort of manipulation is experimentation rather than modeling, sensu stricto. (Step 2-M: here the model is mathematically manipulated.)

The third step concerns the model-data relation, in particular the way that the model is used to explain and increase understanding of the structures and processes of nature, represented as data. In order for the model, a part of the theory, to be applied, it is important to note that the data must also be prepared before it can be made to meet theory. That is, the theoretical assumptions frame how data are to be collected from the chosen structures and processes, and understood (see Kuhn, 1970 on theoryladenness of observation). Thus, there are two kinds of places in which theory imposes itself on data: (1) it strongly determines the form and content of the data [this is a partial denial of thesis (2T) above] and (2) it establishes the relation between itself and the data-i.e. it influences how the data actually bears on the theory as well as how the theory explains the data [this is a partial denial of thesis (3T) above]. In short, theory imposes itself on the data. And, since nature is seen as the cause of the data, theory also imposes itself on nature. (Step 3-E: here the model explains.)

The fourth step pertains to how nature itself, at the end of the modeling process, is interpreted. This step has been completed when the theory-driven understanding of data and nature is considered objective and theory-independent. The historical process of imposing theoretical assumptions onto nature through the modeling process has been forgotten by the end of step 4 and the theoretical assumptions regarding ontology are now understood as really existing in, and as being causally efficacious of, nature. The ontology is now considered intrinsic to the system and is used as mere background against which future scientific investigations are done. (Step 4-O: here the theoretical assumptions about ontology, carried by the model, are objectified.) Below I will present an alternative step 4, pluralize (Step 4-Pl), in which different perspectives, with their respective theoretical assumptions, are explicitly compared and hence objectification and forgetting are avoided.

Note that the process of theoretical imposition occurs throughout the entire four-step process. Although the third step is where the actual imposition on nature occurs, the first two steps are crucial framing steps and the last step is where the consequences are expressed. Let me now turn to the analysis, using the SMEO account, of Turelli's and Frank's respective models of CI evolution.

\section{Michael Turelli's and Steve Frank's models of parasite- mediated CI evolution}

Using my SMEO account, I will now explore two different models of the evolution of CI. Turelli's Fisherian model employs classic population genetic methodology. It appeals to intracellular bacterial density and genetic pleiotropy as the operative mechanisms for the correlational selection that supports CI evolution. Frank's Wrightian model uses newly developed quantitative genetic techniques. This model fundamentally concerns kin selection as the key mechanism for the evolution of CI. There is a further feature of both models that makes them readily 
comparable - they both specify conditions for increase of CI rather than dynamical equations of CI evolution. Before turning to the models, presenting some background information on the Wolbachia system would be useful.

\subsection{Background}

The Wolbachia bacteria is an obligatory endosymbiont found in many arthropod species (16-77\% of insect species sampled, Werren et al., 1995; Jeyaprakash and Hoy, 2000; Stevens et al., 2001), which has evolved a number of strategies for affecting the reproduction of its hosts. Wolbachia is found in the cytoplasm of the somatic and, primarily, reproductive tissues of its host. It can (1) induce parthenogenesis, (2) feminize genetic males, making them functional females, or (3) cause cytoplasmic incompatibility (CI) (e.g. O'Neill et al., 1997; Werren, 1997). As a non-nuclear cytoplasmic parasitic element with little horizontal transmission, it is passed on vertically - through host reproduction-from infected mothers to their ova. Therefore, all the effects on the hosts have evolved, from the parasite's "point of view," to increase, as much as possible, the relative amount of infected cytoplasm in each subsequent generation of hosts. In the case of the third strategy, CI, sperm affected through chromosomal imprinting by the father's Wolbachia (henceforth "infected sperm") that fertilizes uninfected ova very often leads to a zygote that does not develop (Breeuwer and Werren, 1993; Giordano et al., 1995; Turelli and Hoffmann, 1995; Hoffmann and Turelli, 1997; Werren and O'Neill, 1997). All other crosses are viable. Infected sperm can thus be thought of as having the function of destroying competing uninfected (by that Wolbachia strain) cytoplasm in the population.

Wolbachia thus causes three kinds of losses in cases of CI: (1) there is an "infection fecundity cost" such that females with the infection inherently produce fewer ova than females without Wolbachia; and, when infected sperm fertilizes ova either without any Wolbachia, or with a different Wolbachia strain, there is lost offspring from both the (2) infected father's and (3) uninfected (with that strain) mother's "point of view." How exactly should we understand the nature of, and relations among, these three kinds of losses? The two models I turn to provide distinct interpretations. For Turelli, CI evolves as a correlated effect, mediated by genetic pleiotropy or bacterial density, on other components of female fitness, such as female fecundity. He defines and measures fitness always with respect to individual females; losses of type (2) accrue, as losses, to females uninfected with the CI-causing Wolbachia strain. For Frank, on the other hand, CI evolves as an outcome of selection on kin groups. Losses of type (2) are interpreted as gains, via infected fathers, to the infected kin group. In Frank's view, infected males, which do not transmit Wolbachia, act like a warrior caste for infected kin groups. Turelli and Frank do agree on the recipients of losses of types (1) and (3) - infected and uninfected females, respectively. However, one way of seeing the key difference between them is that they measure and interpret, materially and conceptually, type (2) losses in distinct ways, commensurate with the perspectives that they each adopt.

\subsection{Turelli's model}

In what follows, I will outline parts of Turelli's (1994) model using my SMEO account. As we will see, despite his admission that CI evolution offers convincing evidence for Phases II and III of Wright's SBT (Coyne et al., 1997, p. 661), Turelli employs Fisherian assumptions.

\subsubsection{Step $1-S$ in Turelli's model}

There are two particularly strong Fisherian theoretical assumptions, which allow Turelli to set up his model: the absence of direct selection on Wolbachia traits in sperm, and the general absence of kin selection. With respect to the first, in imagining actual biological selective forces on the parasite, Turelli considers sperm evolutionary dead-ends for Wolbachia. There is almost never any paternal transmission of Wolbachia and hence no direct selection on the Wolbachia trait of affecting sperm to express CI. Put differently, any variance in the Wolbachia genome for the trait of CI (a trait which is necessarily expressed only in the combination of infected sperm $\times$ uninfected ovum fertilization, a combination which leads to no or significantly fewer offspring relative to any of the other combinations, depending on the severity of CI) cannot be effectively selected upon and hence the realized heritability is zero. From an evolutionary point of view, selection without transmission is dynamically and kinematically ineffectiveit does not cause any changes of gene frequencies (e.g. Arnold and Wade, 1984; Hartl and Clark, 1989).

The further theoretical assumption that Turelli makes is that there is no kin structure or kin selection. He tends to assume panmixia, that is, random mating within a large population. And even when he considers population structure in special cases, he ignores relatedness. According to Turelli, fitness in structured populations accrues to individuals and not to kin groups. Thus, there can be no indirect "transmission through the sperm" either, in the sense of kin selection. In cases with kin selection, considered by Frank, destruction of uninfected ova, by infected sperm, increase the fitness of (also related) hosts with the same Wolbachia strain as the infected sperm through, for example, increased resource (e.g. space and food) allocation. However, Turelli assumes that this does not occur and, in the set up of his model, has no variables and parameters (henceforth "terms") that could index kin structure.

\subsubsection{Step 2-M in Turelli's model}

Turelli's mathematical manipulation is intricate and subtle. Here I will only emphasize those aspects of step 2$\mathrm{M}$ pertinent to his ontology and which can be directly 
contrasted with Frank's model. I should also state that the initial equations of the model [Eqs. (1) and (2)], which arise from the setup in step 1-S, I first address here in step 2-M. These initial equations, after all, serve as the bridge between the two steps.

Turelli considers the fitness function for the evolution of CI when there is some level of incompatibility (i.e. the Wolbachia variants are not completely compatible; 1994, pp. 1504-1505):

$p_{i, t+1}=p_{i, t} F_{i}\left(1-\mu_{i}\right) \bar{H}_{i} / \bar{W}$.

Here, $F_{i}$ is the fecundity of strain $i$ relative to strain 0 (uninfected females), $\mu_{i}$ is the fraction of uninfected ova produced by infected females of strain $i$ (i.e. this is a measure of the lack of fidelity of maternal transmission), $p_{i, t}$ is the frequency of strain $i$ in generation $t$, and $\bar{W}$ is the mean fitness. This is a well-known population genetic form of selection on a haploid, uniparentally inherited gene (Hartl and Clark, 1989; Wade et al., 2001), wherein $F_{i}(1-$ $\left.\mu_{i}\right) \bar{H}_{i} / \bar{W}$ is the relative fitness of strain $i$. Note the absence of any term tracking kin structure. The term $\bar{H}_{i}$ is defined-relative to each Wolbachia strain $i$, where $i=1$, $2,3, \ldots, n$, where $n$ is the total number of partially incompatible strains - as (Turelli, 1994, p. 1504):

$\bar{H}_{i}=p_{1} H_{i 1}+p_{2} H_{i 2}+\cdots+p_{n} H_{\text {in }}+q H_{i 0}$.

Here, $H_{i j}$ is the relative hatch rate from fertilizations of ova from mothers infected with strain $i$, by sperm from fathers infected with strain $j$, and for which $0 \leqslant H_{i j} \leqslant 1$ indicates a corresponding inverse level of CI (e.g. $H_{i j}=0$ indicates complete incompatibility), $p_{i}$ is the frequency of strain $i$, and $q$ is the frequency of uninfected hosts. Turelli does not add the $H_{i 0}$ term to the $q$, but since he ultimately sets $H_{i 0}=$ $H_{i i}=1$ (1994, p. 1504), I believe that he should include it and, to be consistent and explicit, I add it. Note that total $\mathrm{CI}$ in the population, relative to each strain, changes both when the frequency of different strains changes, and when each $H_{i j}$ changes.

Given the fitness function of Eq. (1), Turelli arrives at a general result for the nature of the term relations necessary for a partially incompatible rare variant (type 1) to increase in frequency in a population with another Wolbachia strain, ${ }^{5}$ when genetic variance in parasites, not hosts, is considered. The condition for increase of a rare variant that he derives is the following (1994, p. 1504):

$F_{1}\left(1-\mu_{1}\right)\left(1-p_{2} s_{h 12}\right)>F_{2}\left(1-\mu_{2}\right)$.

Terms are defined as stated above, and $s_{h i j}$ is $1-H_{i j}$ (i.e. $s_{h i j}$ is a measure of cytoplasmic incompatibility, with 1 representing complete incompatibility and 0 representing complete compatibility). Eq. (3) can be described verbally as follows:

\footnotetext{
${ }^{5}$ In Turelli's explicit model, types 1 and 2 refer to different Wolbachia strains. However, the arguments and conclusions for cases concerning an infected type and an uninfected type (i.e., one without any Wolbachia) are qualitatively similar to those pertaining to Eqs. (3) and (4) analyzed below.
}

If parasite variants are partially incompatible, a new variant will increase when rare only if it increases the 'effective fecundity' [i.e., $F_{1}\left(1-\mu_{1}\right)$ ] of infected females enough to offset the progeny it loses through incompatibility with the infected males already present [i.e., $\left(1-p_{2} s_{h 12}\right)$ ] (see condition 8 [Eq. (3)]). (Turelli, 1994, p. 1509)

Recall that, according to Turelli, there is neither direct selection on sperm nor kin selection. According to Eq. (3), a Wolbachia variant (type 1) increasing levels of CI-that is, decreasing $H_{21}$ and increasing $s_{h 21}$-will increase in proportion in the population of type 2 only if it increases "effective fecundity" sufficiently. Note that $s_{h 21}$ does not appear in the right-hand side of Eq. (3). Therefore, changing CI caused by type 1 (i.e. $s_{h 21}$ ) independently of every other term is causally ineffective in changing the population frequency of type 1 . There is no direct selection on CI. For CI to increase, ${ }^{6}$ it has to be positively correlated with effective fecundity. This is the strong and main conclusion Turelli reaches (see pp. 1509-1510).

Although Turelli emphasizes panmixia, there is an interesting way that population structure can be immediately imported into his model without having to change the parameterization. In Eq. (3) there is a missing and causally ineffective parenthesis on the right-hand side, $\left(1-p_{1} s_{h 21}\right)$. This term is absent because the infected variant is so rare (i.e. $p_{1}$ is very close to 0 ), that its sperm is effectively absent in the panmictic population and hence does not produce a cost to the resident type 2 in terms of unviable type 2 ova fertilized by type 1 sperm. However, with population structure, type 1 could have a large enough frequency locally such that the parenthesis on the right-hand side could no longer be ignored. It would capture (and explain) a causally relevant process. There would now be direct selection on CI caused by type 1 . We would have:

$F_{1}\left(1-\mu_{1}\right)\left(1-p_{2} s_{h 12}\right)>F_{2}\left(1-\mu_{2}\right)\left(1-p_{1} s_{h 21}\right)$.

This development of the model stems from taking seriously a Wrightian theoretical assumption, the existence of population structure.

It is noteworthy that Turelli does write Eq. (4) when he discusses the case of parasite variants that lower effective fecundity [i.e. $\left.F_{1}\left(1-\mu_{1}\right)\right]$. As he rightly claims, such variants can still increase in frequency if they manage to cause sufficiently large losses of type 2 eggs (i.e, $s_{h 21}$ is large) and if they are sufficiently common (i.e. $p_{1}$ is sufficiently different from 0 ). This will only occur with population structure - the initially rare type 1 will now "be able to spread deterministically once it has become established in a

\footnotetext{
${ }^{6}$ There is a lack of clarity in Turelli's article regarding exactly which property of CI is to be explained: level of CI (i.e., $s_{h i j}$ ) or gene frequency of a CI strain (i.e., $p_{i}$ ), or both. His population genetic modeling practices suggest gene frequency, but the citation immediately above implies level of CI. Frank, on the other hand, clearly differentiates the two phenomenahe provides two separate conditions for increases of each (Frank, 1997, p. 329, his Eqs. (3) and (4), respectively).
} 
local population." (1994, p. 1505, emphasis mine). Its establishment occurs through stochastic processes in small populations. ${ }^{7}$ After such a stochastic event increasing its frequency $\left(p_{1}\right)$ sufficiently, type 1 can now deterministically invade the population since females of type 2 , despite still having relatively higher effective fecundities than females of type 1 [i.e. $F_{2}\left(1-\mu_{2}\right)>F_{1}\left(1-\mu_{1}\right)$ ], lose too many offspring through fertilizations with males of type 1 [i.e. $\left(1-p_{1} s_{h 21}\right)$ $\left.\ll\left(1-p_{2} s_{h 12}\right)\right]$.

By appealing to population structure, is Turelli endorsing Wrightian theoretical assumptions in his mathematical manipulation? I believe that he maintains his Fisherian commitments. First, Turelli is motivated to explore Eq. (4), as a special and complex case, because he is concerned with the material positive correlation between parasite density and level of incompatibility (1994, p. 1505; see also the "Discussion," in which he argues that this case has to meet a set of specific, even stringent, conditions, 1994, p. 1510). This is a correlation, to which I will return in step 3-E, which is not considered in Eq. (3), the equation he considers more general and common. Furthermore, he never mentions kin structure and kin selection as relevant to even these cases of population structure, a fact which is conceptually puzzling. Thus, given (A) his emphasis on Eq. (3) rather than Eq. (4), (B) his continual denial of the importance of relatedness and kin selection, even in cases with population structure, and, despite, (C) his admission, together with Coyne and Barton, that CI evolution is compatible with Phases II and III of Wright's SBT (1997, p. 661), it seems most plausible to argue that he continues to provide a Fisherian interpretation of a process that, in fact, is more adequately described as Wrightian.

Let me summarize this step of mathematical manipulation. The bulk of Turelli's theoretical framework is Fisherian. Selection acts directly on effective female fecundity and only through correlation on levels of CI. However, when material considerations, such as bacterial density, are taken seriously as special cases, Turelli turns to an alternative mathematical presentation in which population structure with chance is a necessary condition for $\left(1-p_{1} s_{h 21}\right)$ to be causally effective. Even then, he does not refer to kin selection.

\subsubsection{Step 3-E in Turelli's model}

In this step, the model is used to explain regularities in the data and, ultimately, nature. Recall that this step is a relation between the mathematical model and data/nature. Turelli's mathematical model, however, does not always consistently explain the material regularities.

For Turelli, the two mechanisms in nature that can mediate the correlations between levels of CI and female effective fecundity are bacterial density and genetic pleiotropy. He mentions bacterial density significantly more

\footnotetext{
${ }^{7}$ See also passing mentions of local populations on pp. 1500, 1502, 1510 of Turelli (1994). Furthermore, the model in Turelli and Hoffmann (1991) explicitly incorporates population structure, see especially p. 441 .
}

often than pleiotropy as a mechanism, so I will focus on the former. Nonetheless, for reasons pertinent to theoretical imposition, I will return to the latter.

Turelli, following earlier empirical work, suggests that an increase in bacterial density has the following correlated effects (see especially discussion on p. 1505): (1) it increases the rate of maternal transmission of the bacteria (1994, pp. 1500, 1505), (2) it decreases maternal fecundity (1994, pp. 1500, 1505), and (3) it increases levels of CI (1994, pp. 1505, 1509-1510). Turelli and Frank do agree on the direction of these causal, material correlations determined by bacterial density. Their respective bacterial density ontologies are congruent. Turelli notes that this data is observed especially in the laboratory, where the effects are exacerbated. On the surface, Turelli's modeling results can explain these patterns. Selection on bacterial density among hosts (and the genetics underlying it) occurs through direct selection on female fecundity and maternal transmission (that is, traits that can actually be transmitted), but not on CI (a trait that cannot be transmitted).

However, the effect of increased bacterial density, which both increases levels of $\mathrm{CI}$ and decreases female fecundity (and increases transmission probability), is related to Turelli's mathematical modeling in a complex manner. Eqs. (3) and (4) serve explanatory functions, and although he places more emphasis on Eq. (3), Turelli does not provide clear motivations for choosing between them. Eq. (3) has an ambiguous explanatory relation to the effects of bacterial density. It explains the correlational selection of higher levels of CI by appealing to a positive correlation, ceteris paribus, between level of CI (i.e. $s_{h j i}$ ) and maternal effective fecundity [i.e. $F_{i}\left(1-\mu_{i}\right)$ ], necessary for a variant, causing both of these, to increase in frequency when rare purely by individual selection in a large panmictic population. Only when increases in bacterial density cause a larger increase in transmission probabilities, $\left(1-\mu_{i}\right)$, relative to the decrease in female fecundity, $F_{i}$, will the mathematical correlation be in the same direction as the material correlation. And this cannot be assumed, a priori, to always be the case. Hence, Eq. (3) allows patterns [e.g. when both $F_{i}$ and $\left(1-\mu_{i}\right)$ increase] that are inconsistent with patterns independently inferred through material (not mathematical) considerations of bacterial density. Eq. (4), on the other hand, is built around the material consideration of bacterial density causing a negative correlation between female effective fecundity and level of incompatibility and, as expected, refers to population structure (but not kin selection). It explains the effects of bacterial density by deviating from Turelli's main set of Fisherian assumptions.

In short, Eq. (3) is consistent with Turelli's theoretical assumptions and we are given a convincing exposition of it. However, it does not explain perfectly the data of the material correlations caused by bacterial density. Conversely, Eq. (4) is inconsistent with the Fisherian assumptions and we are not provided a clear motivation for its relevance to Turelli's overall mathematical modeling 
scheme. It is also considered a special and complex case. However, it does explain the material correlation. Turelli's motivations remain unclear.

\subsubsection{Step 4-O in Turelli's model}

Subsequent to the explanatory act, the model and its components are objectified. Nature is understood as inherently containing the theoretical assumptions. For example, concerning the correlations between effective fecundity and levels of $\mathrm{CI}$, in appealing to genetic pleiotropy as an ubiquitous phenomenon, Turelli can postulate any direction for any correlation. This leaves the regularities in nature open to radical theoretical imposition in that he can postulate, and objectify, any genetic correlation required by his model [e.g. the appropriate correlation needed for Eq. (3)]. For example, Turelli discusses how "Variation in... patterns of pleiotropy may underlie the great variation in levels of unidirectional incompatibility associated with Wolbachia in different taxa: from complete... to nearly undetectable." (1994, p. 1510). The theme of pleiotropy comes up in many of Turelli's articles, suggesting that pleiotropy is central to his research program.

The overall objectified ontology of correlational selection (mediated by bacterial density and pleiotropy) as well as individual-female-centered selection processes due to the lack of direct selection on CI is, in important ways, already present as a complex set of theoretical assumptions in the Fisherian perspective. A researcher gets out of a model what she puts into it. The objectified theoretical assumptions are subsequently taken as background for future work. This further use of the ontology indirectly tests (and, most likely, corroborates) it. In addition, Turelli provides suggestions for further experimentation: "By introducing a novel Wolbachia into a large laboratory population, the prediction that $F(1-\mu)$ should increase might be tested directly." (1994, p. 1510, emphasis mine). This suggestion lends further weight to seeing Eq. (3) as central to Turelli's analysis of $\mathrm{CI}$.

\subsection{Frank's model}

In what follows, I will outline parts of Frank's (1997) model using my SMEO account. Frank is concerned with kin selection and, as we will see, articulates a Wrightian model that explicitly shows its importance to CI evolution.

\subsubsection{Step 1-S in Frank's model}

Frank's article starts with setting up the difficulty of increasing the frequency of $\mathrm{CI}$ by distinguishing between the verbal kin selection models of Hurst (1991) and Rousset and Raymond (1991), and the mathematical individual-based models of Turelli (1994) and Prout (1994). For example, although Hurst's verbal model lacks clarity, he presents an early formulation of the issue at hand:
All of the costs of the spiteful act inflict the hosts not the spiteful symbiont. Under these conditions, as the simple models demonstrate, spite can evolve and can be stable. Cytoplasmic incompatibility can thus be seen as a special case of kin selective spite (Hamilton, 1970, 1971). (Hurst, 1991, p. 276)

Spiteful behavior (e.g. CI caused by infected sperm, in which both the infected sperm and uninfected ovum "lose"), in general, cannot evolve. But it is precisely because the behavior (CI) benefits Wolbachia kin, Hurst argues, that it constitutes a spiteful behavior that can actually evolve. This is a theoretical assumption that stands in stark contrast with Turelli's assumptions, and which Frank avails himself of: "I show, with a formal model, that weak kin interactions are sufficient to explain the observed patterns of incompatibility." (Frank, 1997, pp. 327-328; this sentence is not present on pp. 124-125 of Frank, 1998 - otherwise, the relevant text of Chapter 7 of his 1998 book is practically identical to the 1997 article.) Furthermore, he "assume[s] that population regulation occurs within neighbourhoods." (Frank, 1997, p. 328). The theoretical assumption of population structure makes kin interactions relevant to the model and causally potent in nature.

Frank's model is integrated by Wrightian theory, which is committed to the existence and importance of population structure and its associated concept, kin selection (e.g. Eschel, 1972; Hamilton, 1975; Wade, 1978, 1980, 1985, 1992; Uyenoyama and Feldman, 1980; Lloyd, 1988; Frank, 1994, 1998).

\subsubsection{Step 2-M in Frank's model}

Frank presents the overall model: "The first step is to write a fitness function that describes how biological assumptions influence reproduction." (1997, p. 328). He articulates an explicit relative fitness function of the parasite, which eventually, after mathematical manipulation, allows him to bring in population structure and kin structure as pertinent:

$$
\begin{aligned}
w(x, y)= & {[(1-a-b x)(1-\mu)] /\left[(1-l)^{2}\right.} \\
& +l(1-a-b y)+l(1-l)(1-y)] .
\end{aligned}
$$

This fitness function measures the fitness of a parasite as a function of $x$, which is the continuous trait value of that parasite in the host; $y$, which is the average value of that same trait in neighbors with which the host female interacts; $a$, which is the absolute fitness cost the parasite exerts on every infected female; $b x$, which is the relative fitness cost the parasite has on its host in which $b$ is a kind of cost term; $(1-\mu)$, which is, as in Turelli's model, the transmission rate of the parasite; and $l$, which is the frequency of infection (Frank's $q$, not to be confused with Turelli's $q$, which is the frequency of uninfected types) (all on p. 328). Note that the form of this fitness function, with absolute fitnesses presented in an additive rather than 
multiplicative form, is typical of quantitative genetics (Wade et al., 2001).

It is beyond the scope of this article to discuss these terms in detail. Nonetheless, I will briefly elaborate on two: $b$ and $y$. The term $b$, notes Frank, "is the reduction in the fecundity of an infected female that arises as a correlated trait of the level of incompatibility expressed in males." (1997, p. 328). It is a genetic correlation (with a value between -1 and +1 ) and, as such, does not specify the cost for a focal female or even group of females (i.e. $-b x$ is one component of the infection fecundity cost). Rather, as a correlation coefficient, it specifies the direction and tightness of the correlation between the two traits (i.e. infection fecundity cost and level of CI). I will turn to an ambiguity in the definition of $b$ below.

Frank divides his analysis into three main parts: when $b$ is, respectively, equal to, less than, or greater than 0 . Turelli modeled the second case in Eq. (3) (Frank, 1997, p. 328). ${ }^{8}$ Frank considers the last case the most interesting one since that is the biologically most plausible and likely one (since the increased presence of bacteria will both cause higher infection fecundity costs in the female and higher levels of CI in the male), and is also the one for which it is challenging to explain how selection could increase levels of CI (a challenge both Turelli and Frank must, ultimately, meet).

Let me now turn to $y$. For Frank, the key to the increase in levels of CI is kin selection. In order to reach this conclusion from his formal model, he explicitly uses mathematical techniques to index kin structure of the host population and, thereby, of the parasite population. Recall that $y$ is the average value of the trait in neighbors with which the host female interacts. Frank notes that in differentiating the original fitness function [Eq. (5)] with respect to $x$, under any of the three value ranges for $b$, there will always be some $\mathrm{d} y / \mathrm{d} x$ term, which is "the slope of group phenotype on individual genotype, which is the kin selection coefficient of relatedness [Hamilton's $r$ ]." (Frank, 1997, p. 328). Frank also refers to a "direct fitness" methodology, not actually presented in his 1997 article, which provides the meaning for the $\mathrm{d} y / \mathrm{d} x$ term. He developed this methodology with a co-author (PD Taylor) in a 1996 article entitled "How to make a kin selection model"- - this method is further developed in Chapter 4 of Frank (1998). The interested reader of Frank's (1997) article can refer to the 1996 co-authored article and the 1998 book as a kind of generative "mathematical manual" (rather than, say, a "laboratory manual") in which

\footnotetext{
${ }^{8}$ Frank notes that "Prout and Turelli's formal models do not address the original ideas about selfish parasites and kin selection as an explanation for incompatibility. Their models implicitly assume that there is no population structure and therefore no potential for kin interactions." (1997, p. 327). However, in so far as Turelli adopts Eq. (4), he does accept both population structure and $b>0$, but without appealing to kin structure and kin selection. As we also saw above, how he is able to do this justifiably is genuinely puzzling.
}

strategies for how to build a formal kin selection model can be found:

[the "direct fitness"] method provides an orderly set of tools for studying the multiple pathways by which social interactions influence fitness. More importantly, the evolutionary processes stand out clearly during the analysis, so that the analysis itself enhances our understanding of the problem. (Taylor and Frank, 1996, p. 36)

Thus, $\mathrm{d} y / \mathrm{d} x$ (i.e. $r$ ) explicitly provides the grounds for tracking and mathematically manipulating kin structure.

Frank, unlike Turelli, presents clear conditions for both the increase of the value of the CI trait (i.e. under the respective parameterizations: Turelli's $s_{h i j}$; Frank's $x$, and, eventually, $z$ ) as well as the frequency of infection in the population [i.e. under the respective parameterizations: Turelli's $=p_{i}$; Frank's $=q$, written as $l$ in Eq. (5) above] (Frank, 1997, p. 329). For example, the "condition for selection to favour an increase in the incompatibility trait, $z$, is":

$r q(b+1-q)>b$.

He derives this by finding when $\mathrm{d} w / \mathrm{d} x>0$, where Eq. (5) defines $w$. Frank uses Eq. (6), together with the condition for selection to favor an increase in $q$ [Eq. (4) in 1997, p. 329], to both express the dynamics of change under different values of the terms $b, q, r, z$, and $\mu$, and to find stable local equilibiria of $q$ and $z$ in the overall parameter space.

There is an ambiguity in the relation between $b$ and $r$ in Frank's model - it is not clear that they are, indeed, independent as Frank's analysis requires (e.g. Fig. 2, p. 330, with $b$ and $r$ as orthogonal axes). Here I will merely suggest one possible interpretation for how changes in $r$ could be seen as necessarily changing the value of $b$. Recall that he defines $b$ as "the reduction in the fecundity of an infected female that arises as a correlated trait of the level of incompatibility expressed in males" (1997, p. 328). The notion of "fecundity" is vague: is it a sort of "intrinsic" fecundity (i.e. number of eggs laid by the individual, relative to the uninfected type, as in Turelli's model), or an "extended" fecundity that tracks inclusive fitness (i.e. sum of the relative number of eggs laid by the focal female and her relatives, each individual weighted by $r$ )? Since there are reasonable causal theoretical assumptions underlying the second interpretation, consistent with Frank's Wrightian setup, it cannot be immediately discarded. And, even without further development of the formalisms, it should be clear that the latter interpretation implies that changes in $r$ cause, by definition, changes in $b$, and so we would not be able to vary the two independently of one another, as Frank does. The exact interpretation of $b$, and the causal mechanisms underlying it (including bacterial density as a possibility), are worth detailing in future work.

Ultimately, Frank's investigation is "meant as a rough, qualitative guide to the complex dynamics of the system. 
The main point is that relatedness, $r$, can strongly influence selection of incompatibility." (1997, pp. 329-330, emphasis mine). Frank summarizes his theoretically motivated results in the following fashion:

Prout (1994) and Turelli (1994) implicitly assumed that $r=0$. Given that assumption, it is not surprising that they concluded kin selection does not favour incompatibility. I have shown that the simple condition $r>0$ is sufficient to favour incompatibility when there is no genetic correlation between incompatibility expressed in infected males and reduced fecundity expressed in infected females (the parameter $b=0$ ). When there is a correlation, $b>0$, [i.e., there is a positive infection fecundity cost] kin selection influences incompatibility, but the net selective effect depends on the relative magnitudes of relatedness, $r$, negative effects on female fecundity, $b$, transmission efficiency, $\mu$, and the frequency of infection, $q$ [written as $l$ in Eq. (5) above]. The direction of selection can shift toward higher or lower incompatibility as these factors change in magnitude. (1997, p. 330; this paragraph is not present in Frank, 1998).

Kin selection has a significant effect and, for some ranges of parameter and variable values, can increase both the value and frequency of $\mathrm{CI}$. This is the fundamental conclusion from Frank's mathematical manipulation.

\subsubsection{Step 3-E in Frank's model}

Kin selection has significant explanatory power under Frank's model. Contra Turelli's Eq. (3), Frank's explanatory account appeals to an intuitive positive correlation between "the reduction in the fecundity of an infected female" and "the level of incompatibility expressed in males." (1997, p. 328). Because of his introduction of another variable, $r$, motivated by a rich theoretical (Wrightian) background, Frank suggests: (1) that there is indeed direct selection, as a kin group of parasites (and hosts), on levels of CI and (2) that increased levels of CI are correlated (mathematically and materially) with a decrease in infected female (intrinsic) fecundity. The advantage of increased CI, to the parasite, in this case comes from "the beneficial effects of incompatibility, proportional to the coefficient of relatedness in the neighbourhood." (1997, p. 329). Succinctly put, "the parasite in the male does not reproduce, but can aid related parasites in neighbouring females." (1997, p. 327).

Frank, in emphasizing kin selection from a genetic point of view, rather than correlational selection or population structure with chance, provides a different, but equally theory-laden, explanation of the material selective process of increasing levels of CI. His model includes: (1) theoretical assumptions regarding the ubiquity of population structure and kin structure, as well as (2) mathematical techniques and structures (e.g. the "direct fitness" method of Taylor and Frank, 1996; Frank, 1998; $\mathrm{d} y / \mathrm{d} x=r$ ) that track kin structure and kin selection.
Furthermore, recall that Frank states that "weak kin interactions are sufficient to explain the observed patterns of incompatibility." (1997, pp. 327-328, emphasis mine). Although he never makes the claim that kin interactions are necessary to (all kinds of) selection, given that he does not consider alternative models that leave out kin selection-indeed, in this article and elsewhere (e.g. Frank, 1994, 1998) he seems to consider such alternative models suspect-it does seem that he considers kin selection a necessary component for any complete explanation of evolutionary change. Thus, this is most likely a case of a particular model-based explanatory relation that could be called making the merely explanatorily sufficient explanatorily necessary.

\subsubsection{Step 4-O in Frank's model}

Although Frank tends to stay at an abstract theoretical level in his work, he also sees kin selection as a central and objectively ubiquitous process in nature. His impressive 1998 book continues his research project of articulating the crucial role of kin selection in the process of social evolution.

The example of CI evolution, then, constitutes one of multiple cases (as outlined in Frank, 1998) that kin selection explains, thereby further confirming the Wrightian perspective. It should be noted that although this perspective may very well be the appropriate one for CI evolution, this case also involves theoretical imposition. First, Frank has developed a particular kind of kin selection mathematical model. There are other types of models of kin selection that need to be considered. Second, although this case does very much seem to require the conceptualization and tracking of kin structure and kin selection, it is not clear that this is required in general to explain other cases of social evolution, as Frank's book argues. It may be problematic to objectify kin selection as a component of the general ontology of nature and, thereby, use this form of selection as a background for all further work. Further case-by-case comparative SMEO analyses, such as the present one, of the relevance of the Wrightian perspective for other cases of social evolution are necessary.

\subsection{Step 4-Pl in Turelli's and Frank's models}

This comparative case allows me to introduce step 4Pluralize (4-Pl). The goal of this step is to become aware of the theoretical assumptions of different perspectives. These distinct complexes of theoretical assumptions (e.g. Turelli's and Frank's and, to consider a third alternative, Wade and Stevens, 1994) should be compared for respective strengths and weakness, as well as for shared robustness. In this alternative step, we distance ourselves from commitments to particular perspectives, and, instead, incorporate all the operative perspectives and efforts at modeling. To what kinds of further theorizing and experimenting do different theoretical assumptions point? How are the theories, and 
associated ontologies, related? Are they (1) mutually incompatible, (2) translatable and, furthermore, generalizable, or (3) applicable to different domains of nature [independently of (1) or (2)]? In which step(s) of the SMEO account does theoretical imposition occur in the different perspectives? Pluralizing sets of theoretical assumptions, including ontologies, allows us to be critical of theoretical imposition and its cascading effects in further scientific theorizing and experimentation.

\subsection{On the translatability of the two models}

By comparing the parameterization of the two models, including their respective characterizations of mean fitness, I will argue that the two models are, to a large extent, but not perfectly, mathematically translatable and generalizable. This explicit comparison also allows me to detect the way assumptions are placed into the models and subsequently imposed.

Let us set the two formalizations of relative fitness, $w$, equal to each other. Recall that $F_{i}\left(1-\mu_{i}\right) \bar{H}_{i} / \bar{W}$ is the relative fitness of a parasite in an infected female in Turelli's model, ${ }^{9}$ as expressed in Eq. (1), and that Eq. (5) is relative fitness in Frank's model. For heuristic purposes of simplicity, let us eliminate $\bar{W}$ from both equations. Admittedly, the structure of $\bar{W}$ is different in both equations; however, since they are each a valid measure of mean fitness, they can be interpreted as equal (below I will return to the two different structures of mean fitness). Eliminating $\bar{W}$ we have:

$F_{i}\left(1-\mu_{i}\right) \bar{H}_{i}=(1-a-b x)(1-\mu)$.

The term $(1-\mu)$ is defined identically in both models, so we can also remove it. Furthermore, Frank assumes that there is only one infection strain ${ }^{10}$ and, in that case, $\bar{H}_{i}$ (with

\footnotetext{
${ }^{9}$ Strictly speaking, as we shall see below, the absolute fitness of an infected female, in Turelli's model, also has a $\left(\mu_{i} \bar{H}_{0}\right)$ component: $F_{i}\left[\left(1-\mu_{i}\right) \bar{H}_{i}+\mu_{i} \bar{H}_{0}\right]$. However, to make the mathematical comparison between the two models exact I will only consider the absolute fitness as indicated in Eq. (1) since this is indeed, the absolute fitness for the parasite in the infected female. The $\left(\mu_{1} \bar{H}_{0}\right)$ term, as presented in Table 1, represents the contribution to the fitness of the infected female of her uninfected offspring, which do not, by definition, have the parasite. There is, therefore, an ambiguity in Turelli's definition of absolute fitness: is it measured from the parasite's or the host's point of view?

${ }^{10}$ Frank (pers. comm., August 5, 2005) argues that "the approach I used assumes a continuum of genetically based phenotypes near the equilibrium value, that is, an essentially infinite variety of different genotypes with phenotypes $x$ that vary around the equilibrium $x^{*}$." This is a subtle and important point. I believe that this is consistent with arguing that Frank only appeals to one type of Wolbachia strain in his model, with a significant amount of intra-strain genetic variation. Note that Turelli also assumes that each type of strain has substantial intra-strain genetic variation, but he explicitly appeals to different types of strains which would, under Wrightian assumptions, form individual kin groups and lineages, and, potentially, be mutually incompatible. I will not here further address the fundamental question of how to differentiate inter-strain from intra-strain genetic variation. I will operate under the supposition that Frank assumes the existence of one strain, while Turelli assumes the existence of multiple strains.
}

$i=1$ ) in Turelli's model [Eq. (2)] reduces to 1 , given a normalization assumption he makes regarding $H_{10}$ and $H_{11}$ (Turelli, 1994, p. 1504). We therefore have:

$F_{1}=1-a-b x$.

Recall that Frank's expression, on the right-hand side, is "the focal female's reproductive rate."11 (Frank, 1997, p. 328). Thus, in this case, Turelli's $F$, the relative number of eggs a focal female lays, is identical to Frank's definition of reproductive rate. In cases with more than one strain, the equivalence is absent, since $\bar{H}_{i}$ would no longer be equal to 1 as the different infection strains would have different $H$ 's. However, in these cases, we could no longer justifiably compare the two models because the assumption of only one infection strain is fundamental to Frank's model. So the relative fitnesses are equal under the pertinent assumptions.

Let us now investigate the structure of mean fitness under each model. Although it is unclear how to exactly translate Turelli's $F$ and $H$ terms into Frank's $a$ and $b$ terms under all conditions, it is clear that both use the combinatorial method of tracking frequencies and fitnesses of family level mating types (e.g. Wade, 1980; CavalliSforza and Feldman, 1981; Hartl and Clark, 1989). Since here male and female cytoplasm is being tracked, the inheritance pattern is analogous to a haploid, uniparentally inherited gene. In an important sense, then, the two characterizations of $\bar{W}$ are instantiations of the same general structure and can, thereby, be translated and generalized (Table 1).

A few comments regarding these absolute fitnesses are in order. Note that Frank presents three different types of fitnesses. The fitness of one accruing to $q \times q$ matings is the baseline. As we saw above in Section 3.1, there are three kinds of losses. Frank accounts for each of them. The fitness $(1-a-b y)$ represents "the relative fecundity of infected females in the neighbourhood" (Frank, 1997, p. 328), which involves the infection fecundity cost (type 1 cost). The parasite reduces fecundity due to both a fixed amount in all infected females, $a$, and a relative amount, due to the average value of the level of $\mathrm{CI}$ in males in the neighborhood, $y$, as correlated, $b$, with the trait of reduction in (intrinsic) fecundity of an infected female. The lost mating cost from the point of view of the uninfected female (type 3 cost) is indicated by $(1-y)$; note that if the average value of CI is at its highest in the neighborhood $(y=1)$, the fitness of the uninfected female will be 0 -it will lose all its offspring. The type 2 cost, as discussed above, is included as gains for the kin group of infected types, and is only clear subsequent to the appearance of $r$ after mathematical manipulation, such as in Eq. (6).

Turelli, however, only presents two kinds of fitnesses, for females with $\left(W_{11}=W_{12}\right)$, and without $\left(W_{21}=W_{22}\right)$, the

\footnotetext{
${ }^{11}$ For Frank, however, this is true only under the "intrinsic" fecundity interpretation of $b$.
} 
Table 1

Comparison of absolute fitnesses of mating types, derived from their respective models [Eqs. (1) and (5), respectively] ${ }^{\mathrm{a}}$

\begin{tabular}{|c|c|c|c|c|}
\hline \multicolumn{2}{|c|}{ Mating-type femalexmale (frequency) } & \multirow{2}{*}{$\begin{array}{l}\text { Absolute fitnesses of mating } \\
\text { types (General) }\end{array}$} & \multirow{2}{*}{$\begin{array}{l}\text { Absolute fitnesses of mating } \\
\text { types (Frank) } \\
1-a-b y\end{array}$} & \multirow{2}{*}{$\begin{array}{l}\begin{array}{l}\text { Absolute fitnesses of mating types } \\
\text { (Turelli) }\end{array} \\
F_{1}\left[\left(1-\mu_{1}\right) \bar{H}_{1}+\mu_{1} \bar{H}_{0}\right]\end{array}$} \\
\hline$p \times p$ & $\left(p^{2}\right)$ & & & \\
\hline$q \times p$ & $(p q)$ & $W_{21}$ & $1-y$ & $F_{0}\left[\bar{H}_{0}\right]$ \\
\hline$q \times q$ & $\left(q^{2}\right)$ & $W_{22}$ & 1 & $F_{0}\left[\bar{H}_{0}\right]$ \\
\hline
\end{tabular}

Note that $p(=l$ in Frank's model) is the frequency of the infection. Mean fitness is derived by multiplying each frequency by each fitness and, subsequently, factoring and otherwise simplifying to get Eqs. (9) and (10).

${ }^{a}$ The absolute fitnesses for Frank's model can be derived by direct inspection from the denominator of Eq. (5). In the case of Turelli, however, the derivation is more subtle. The general mean fitness equation for one locus with two alleles is $\bar{W}=W_{11} p^{2}+W_{12} p q+W_{21} p q+W_{22} q^{2}$. And if we use the absolute fitnesses indicated in the table, weighted by the mating type gentoype frequencies, we get Turelli's mean fitness function, Eq. (9) below. The two types of "cytoplasmic heterozygote" $(p \times q)$ mating types will not have equal fitness (i.e., $\left.W_{21} \neq W_{12}\right)$ because, according to Turelli, the female cytoplasms are different. However, Turelli sets $W_{21}=W_{22}$, which is a problem that I will turn to below.

infection. The problem is that he assigns the same fitness to the $q \times p$ and $q \times q$ matings: $W_{21}=W_{22}$. This amounts to ignoring costs of types 2 and 3 , since there is no fitness reduction, of any sort, through lost offspring. Perhaps these costs could be interpreted as already present in the $H_{i j}$ terms of Eq. (2), but then the loss is illegitimately averaged out across mating types and associated only with cytoplasmic types. That is, he is averaging pairs of family fitnesses, those with the same female type (either infected or uninfected), into a single fitness for each cytoplasmic type. This way of averaging is similar to calculating the average fitness of a female over two environments, only here, the frequency of the environments (i.e. the male types) is itself a function of the frequency of Wolbachia infection in the population. That is, the relevant environment is created by the mating activity of the hosts. One way he could incorporate these costs would be by presenting different $H$ 's for each mating type and not just, as he has it now, for female cytoplasmic type. Despite this, he does include the type 1 cost in the $F$ s. $F_{0}$ is the fecundity of uninfected females, presumably equal to 1 (see Turelli, 1994, pp. 1502, 1504), and $F_{1} \leqslant F_{0}$.

Given the absolute fitnesses in Table 1, these are the two expressions for $\bar{W}$ :

(1) Turelli's [Eq. (7a) in 1994, p. 1504]: ${ }^{12}$

$$
\bar{W}=p_{1}\left[F_{1}\left(1-\mu_{1}\right) \bar{H}_{1}+F_{1} \mu_{1} \bar{H}_{0}\right]+q F_{0} \bar{H}_{0} .
$$

(2) Frank's [denominator of Eq. (1) in 1997, p. 328; Eq. (5) above]:

$$
\bar{W}=(1-l)^{2}+l(1-a-b y)+l(1-l)(1-y) .
$$

\footnotetext{
${ }^{12}$ I have made two changes. Although Turelli also includes a term for $p_{2}$, of the same form as that of $p_{1}$ (except that the 1's are replaced by 2's), I have ignored it since I am only considering one infection strain, in order to make the comparison with Frank's model explicit. Also, Turelli normalizes $F_{0}$ by setting it equal to one and thereby omitting it from Eq. (9). I have added, to be consistent and explicit, $F_{0}$ to the $q$ term.
}

In Turelli's case, $\bar{W}$ does not track relatedness. It is interesting to note here that with the theoretical assumptions of population structure and kin structure, relatedness would be "hidden in" the term, $\bar{H}_{i}$, as well as in its component terms, $H_{i j}$ (Michael Wade, pers. comm.). This is because $\bar{H}_{i}$, as expressed in Eq. (2), depends upon the interaction of at least two infected host types. The respective $H_{i j}$ 's of these types would, in fact, change as a consequence of any change in the structured distribution of matings - that is, of change of relatedness relations. In other words, if we did assume population structure and kin structure, each $H_{i j}$ and $\bar{H}_{i}$, would have to be made a function of new kinds of terms that track such structure, almost certainly including Hamilton's $r$. However, if panmixia in large populations is assumed, as Turelli generally does, then neither relatedness nor population structure are pertinent: we can average across a large panmictic population to get the $H_{i j}$ terms. Thus, for cases when $\bar{H}_{i}$ is not one (i.e. more than one infection strain), Turelli's Fisherian assumption of panmixia allows him to solely use averaged and primitive $H_{i j}$ 's to get $\bar{H}_{i}$.

On the other hand, as we saw above, Frank's modeling of $\bar{W}$ explicitly indexes kin structure. Mean fitness is a consequence of interactions between individuals and their neighbors. But neighbors are often related in Frank's model, and Taylor and Frank's modeling methodology discussed above allows us to explicitly define $r$ in terms of such interactions (i.e. $r$ is "the slope of group phenotype on individual genotype"; Frank, 1997, p. 328). This is consistent with his Wrightian theoretical assumptions.

Are the models, then, mathematically translatable and generalizable? Although there is not an absolutely unequivocal answer to this question, I believe that, to a large extent, they are. First, under the assumption of just one infection strain, the relative fitnesses, in both models, of a parasite in an infected female are equal. It is not possible to say how the models relate under the assumption of more than one strain, since Frank's model assumes only one infection strain (footnote 10 above). Second, they both employ the family level mating type method to derive mean 
fitness. However, they do contain different terms whose complete and exhaustive mapping relations await further exploration. To a large extent, the models are comparable and commensurable mathematically.

\section{Conclusions: theoretical imposition, the SMEO account, and the Fisherian-Wrightian controversy}

In this article, I have shown that modeling is not ontologically innocent. We do not simply apply a model to theory-independent data acquired from an autonomous nature. First of all, the data are shaped by our theory (e.g. family level selection in chickens). Second, different theories impose different ontologies onto a somewhat flexible and indeterminate nature (e.g. Turelli's and Frank's respective ontologies stemming from the Fisherian and Wrightian perspectives).

My focus on theoretical imposition, as tracked in the SMEO account, is not meant as a denial of the utility and importance of the view represented by theses (1T)-(3T) in the first section, which, among other points, emphasizes theory-independent data. Defending a view is not tantamount to denying its alternative. Both can be true in a variety of ways. But specifying under which conditions this could happen requires further conceptual work. In the same way that we need to resolve how two different biological perspectives, the Fisherian and Wrightian ones, relate, we need to resolve how the philosophical views of nature-independence and theoretical imposition relate. Are they translatable and generalizable? Are they each true under different assumptions or in different domains? Further investigation is required.

Here I hope to have convinced the reader that theoretical imposition clearly exists in the case of modeling CI evolution. I have argued that differences between the two models in the mathematical presentation of the models, explanations, and objectified ontologies stem neither from differences in mathematical method nor the employed data, but from differences in the theoretical assumptions, especially regarding ontology, already present in the respective perspectives. Given this, which general lessons pertinent to the controversy between Fisherian and Wrightian perspectives can be drawn from the results of my analysis of this modeling case? I will answer this question in five steps, with a sixth step added to discuss the general applicability of my SMEO model to other cases:

1. This modeling case is a "controlled conceptual experiment" in the sense that two factors, process studied and mathematical methods, are, roughly, constant across the two models, whereas the theoretical assumptions, especially regarding ontology, vary. Differences in the conclusions inferred from the models, therefore, stem mainly from differences in the theoretical assumptions. These theoretical assumptions are imposed.

2. One way to track this theoretical imposition is through my SMEO account, which clearly separates a variety of steps of the modeling process. A careful analysis of the mathematics (step 2-M) is particularly useful for following the theoretical imposition.

3. At least in this modeling case, mathematical equivalence or compatibility (recall Wade and Goodnight's observation that "the mathematical details of these theories are largely in agreement"; 1998, p. 1537) is not evidence for ontological equivalence or compatibility. Radically different ontologies can be modeled using multiple compatible mathematical models, with different conclusions being reached for each model.

4. At least in this modeling case, as well as in the example of family level selection of chickens, similar data can be interpreted in radically different ways under distinct perspectives. Equivalence or similarity of data, thus, is also not evidence for ontological equivalence or compatibility. Empirical representations of radically different ontologies can stem from similar data, with different conclusions being reached under each interpretation of that data.

5. This modeling case is one in which there is mathematical equivalence and significant overlap, if not identity, in experiments and observations (e.g. effects of bacterial density) appealed to by the different parties. Nevertheless, deep differences between the Fisherian and Wrightian perspectives remain. These perspectival differences concern the theoretical assumptions regarding ontology - that is, hierarchical structures, as well as different kinds of processes and causes, in nature. In order to continue a comparative analysis of these perspectives, with the hope of ultimately overcoming, or at least understanding, the tensions between them, it will not be sufficient to explore only their respective mathematical models or data. I suggest that we embark on a close examination of the ontological assumptions, and the way they are imposed through the modeling process, in these perspectives. My SMEO account allows us to do this for cases of mathematical modeling, such as this one. A concerted and clear effort must also be made to engage in step 4-Pl, the step of the modeling process in which the ontologies are explicitly compared for the purpose of trying to understand whether they are, (1) mutually incompatible, (2) translatable and generalizable, or (3) applicable to different domains of nature. I do not believe that an answer can yet be provided, however, regarding the final and definitive status of the relation between multiple ontologies in the controversy between Fisherian and Wrightian perspectives. Further exploration of this relation is required.

6. The SMEO account, as I have shown here, allows us to track theoretical imposition by multiple perspectives examining a single structure or process; it can also be used to analyse cases, possibly more numerous, of theoretical imposition by a single perspective. In order to be applied elsewhere, my account has to be tested and, possibly, changed since, as I pointed out in footnote 4 above, it is very likely that it contains biases 
stemming both from my own views about modeling and from using the Fisherian-Wrightian controversy as my data point. Put differently, a robustness analysis (sensu Levins, 1966; Wimsatt, 1981) of the SMEO account remains undone. In the process of doing such an analysis, (variants of) the account would be used to track theoretical imposition in a multitude of other cases; of course, the jury is also still out regarding the relation among multiple ontologies in other cases involving multiple perspectives. In order to reach conclusions, pertinent to both biology and philosophy of science, about theoretical imposition by single and multiple perspectives, as well as relations among ontologies in a variety of controversies involving multiple perspectives, further investigation is needed.

\section{Acknowledgements}

Discussions with audience members at a presentation at the Vrije Universiteit (Free University) of Amsterdam, including Marcel Boumans, Henk de Regt, Sabina Leonelli, Cor van der Weele, and Arno Wouters, as well as at a presentation at the Instituto de Investigaciones Filosóficas in Mexico City, including Axel Barceló, Roy Brand, Carlos Pereda, and Sergio Martínez, were extremely productive. I am very grateful to Roy Brand, Steve Crowley, Mark Detweiler, Steve Frank, Vivette García Deister, Elihu Gerson, Peter Godfrey-Smith, Benjamin Kerr, Hervé Kieffel, Sabina Leonelli, Elisabeth Lloyd, Sergio Martínez, Narisa Murray, Amir Najmi, Lourdes Ortiz, Susan Oyama, Alba María Ruibal, Francisco Vergara-Silva, Michael Wade, Günter Wagner, and two anonymous reviewers for incisive comments on earlier drafts. I would particularly like to acknowledge the constant inspiration and efforts of my teachers and mentors, Elisabeth Lloyd and Michael Wade, both of whom are as much philosopher-biologists as biologistphilosophers.

\section{References}

Arnold, S.J., Wade, M.J., 1984. On the measurement of natural and sexual selection: theory. Evolution 38, 709-719.

Bourke, A.F.G., Franks, N.R., 1995. Social Evolution in Ants. Princeton University Press, Princeton, NJ.

Breeuwer, J.A.J., Werren, J.H., 1993. Cytoplasmic incompatibility and bacterial density in Nasonia vitripennis. Genetics 135, 565-574.

Caballero, A., Keightley, P.D., Hill, W.G., 1991. Strategies for increasing fixation probabilities of recessive mutations. Genet. Res. 58, 129-138.

Cartwright, N., 1983. How the Laws of Physics Lie. Oxford University Press, Oxford, UK.

Cartwright, N., 1999. Models and the limits of theories: quantum Hamiltonians and the BCS model of superconductivity. In: Morgan, M., Morrison, M. (Eds.), Models as Mediators. Perspectives on Natural and Social Sciences. Cambridge University Press, Cambridge, UK, pp. 241-281.

Cavalli-Sforza, L.L., Feldman, M.W., 1981. Cultural Transmission and Evolution: A Quantitative Approach. Princeton University Press, Princeton, NJ.
Coyne, J.A., Barton, N.H., Turelli, M., 1997. Perspective: a critique of Sewall Wright's Shifting Balance Theory of evolution. Evolution 51, 643-671.

Coyne, J.A., Barton, N.H., Turelli, M., 2000. Is Wright's Shifting Balance Process important in evolution? Evolution 54, 306-317.

Craig, D.M., Muir, W.M., 1996. Group selection for adaptation to multiple-hen cages: beak-related mortality, feathering, and body weight responses. Poult. Sci. 75, 294-302.

Crozier, R.H., Pamilo, P., 1996. Evolution of Social Insect Colonies. Oxford University Press, New York.

Endler, J.A., 1986. Natural Selection in the Wild. Princeton University Press, Princeton, NJ.

Eschel, I., 1972. On the neighbor effect and the evolution of altruistic traits. Theor. Popul. Biol. 3, 258-277.

Falconer, D.S., Mackay, T., 1996. Introduction to Quantitative Genetics. Longman, Essex, UK.

Frank, S., 1994. Kin selection and virulence in the evolution of protocells and parasites. Proc. R. Soc. London Ser. B 258, 153-161.

Frank, S., 1997. Cytoplasmic incompatibility and population structure. J. Theor. Biol. 184, 327-330.

Frank, S., 1998. Foundations of Social Evolution. Princeton University Press, Princeton, NJ.

Frank, S., Slatkin, M., 1992. Fisher's fundamental theorem of natural selection. Trends Ecol. Evol. 7, 92-95.

Gayon, J., 1998. Darwinism's Struggle for Survival. Heredity and the Hypothesis of Natural Selection. Cambridge University Press, Cambridge.

Giordano, R., O'Neill, S.L., Robertson, H.M., 1995. Wolbachia infections and the expression of cytoplasmic incompatibility in Drosophila sechellia and D. mauritiana. Genetics 140, 1307-1317.

Goodnight, C.J., Wade, M.J., 2000. The ongoing synthesis: a reply to Coyne, Barton, and Turelli. Evolution 54, 317-324.

Griesemer, J.R., 1990. Modeling in the museum: on the role of remnant models in the work of Joseph Grinnell. Biol. Philos. 5, 3-36.

Hamilton, W.D., 1970. Selfish and spiteful behaviour in an evolutionary model. Nature 228, 1218-1220.

Hamilton, W.D., 1971. Selection of selfish and altruistic behavior in some extreme models. In: Eisenberg, J.F., Dillon, W.S. (Eds.), Man and Beast: Comparative Social Behavior. Smithsonian Inst Press, Washington, DC, pp. 57-91.

Hamilton, W.D., 1975. Innate social aptitudes in man: an approach from evolutionary genetics. In: Fox, R. (Ed.), Biosocial Anthropology. Wiley, New York, pp. 133-155.

Hartl, D.L., Clark, A.G., 1989. Principles of Population Genetics. 2nd ed. Sinauer Associates, Inc., Sunderland, MA.

Hoffmann, A.A., Turelli, M., 1997. Cytoplasmic incompatibility in insects. In: O'Neill, S., Hoffmann, A.A., Werren, J.H. (Eds.), Influential Passengers. Inherited Microorganisms and Arthropod Reproduction. Oxford University Press, Oxford, UK, pp. 42-80.

Hurst, L.D., 1991. The evolution of cytoplasmic incompatibility or when spite can be successful. J. Theor. Biol. 148, 269-277.

Jeyaprakash, A., Hoy, M., 2000. Long PCR improves Wolbachia DNA amplification: $w s p$ sequences found in $76 \%$ of sixty-three arthropod species. Insect Mol. Biol. 9, 393-405.

Kuhn, T.S., 1970. The Structure of Scientific Revolutions, second ed. University of Chicago Press, Chicago.

Levins, R., 1966. The strategy of model building in population biology. Am. Sci. 54, 421-431.

Levins, R., 1968. Evolution in Changing Environments. Princeton University Press, Princeton, NJ.

Levins, R., Lewontin, R.C., 1985. The Dialectical Biologist. Harvard University Press, Cambridge, MA.

Lewontin, R., 1974. The Genetic Basis of Evolutionary Change. Columbia University Press, New York.

Lloyd, E.A., 1988. The Structure and Confirmation of Evolutionary Theory. Princeton University Press, Princeton, NJ.

Lloyd, E.A., 2000. Units and levels of selection: an anatomy of the units of selection debates. In: Singh, R., Krimbas, C., Paul, D., Beatty, J. 
(Eds.), Thinking About Evolution: Historical, Philosophical, and Political Perspectives. Harvard University Press, Cambridge, MA, pp. 267-291.

Michod, R.E., 1999. Darwinian Dynamics. Princeton University Press, Princeton, NJ.

Morgan, M.S., Morrison, M. (Eds.), 1999. Models as Mediators. Perspectives on Natural and Social Science. Cambridge University Press, Cambridge, UK.

Morrison, M., 2000. Unifying Scientific Theories. Physical Concepts and Mathematical Structures. Cambridge University Press, Cambridge, UK.

Muir, W.M., 1996. Group selection for adaptation to multiple-hen cages: selection program and direct responses. Poult. Sci. 75, 447-458.

Muir, W.M., 2005. Incorporation of competitive effects in forest tree or animal breeding programs. Genetics 170, 1247-1259.

Neyman, J., Park, T., Scott, E.L., 1956. Struggle for existence. The Tribolium model: biological and statistical aspects. In: Neyman, J. (Ed.), Proceedings of the Third Berkeley Symposium on Mathematical Statistics and Probability, vol. 4. University of California Press, Berkeley, CA, pp. 41-79.

O’Neill, S., Hoffmann, A.A., Werren, J.H. (Eds.), 1997. Influential Passengers. Inherited Microorganisms and Arthropod Reproduction. Oxford University Press, Oxford.

Oyama, S., 1985. The Ontogeny of Information. Developmental Systems and Evolution. Cambridge University Press, Cambridge, UK Reprinted by Duke University Press, 2000.

Prout, T., 1994. Some evolutionary possibilities for a microbe that causes incompatibility in its host. Evolution 48, 909-911.

Provine, W.B., 1986. Sewall Wright and Evolutionary Biology. University of Chicago Press, Chicago.

Rousset, F., Raymond, M., 1991. Cytoplasmic incompatibility in insects: why sterilize females? Trends Ecol. Evol. 6, 54-57.

Skipper, R.J., 2002. The persistence of the RA Fisher-Sewall Wright controversy. Biol. Philos. 17, 341-367.

Smith, B.C., 1996. On the Origin of Objects. MIT Press, Cambridge, MA.

Sober, E., 2003. Parsimony. In: Sarkar, S., Pfeifer, J. (Eds.), The Philosophy of Science. An Encyclopedia. Routledge Press, London, UK URL $=\langle$ http://philosophy.wisc.edu/sober/pars.PDF $\rangle$.

Stevens, L., Giordano, R., Fialho, R.F., 2001. Male-killing, nematode infections, bacteriophage infection, and virulence of cytoplasmic bacteria in the genus Wolbachia. Annu. Rev. Ecol. Systemat. 32, 519-545.

Taylor, P.D., Frank, S.A., 1996. How to make a kin selection model. J. Theor. Biol. 180, 27-37.

Turelli, M., 1994. Evolution of incompatibility-inducing microbes and their hosts. Evolution 48, 1500-1513.

Turelli, M., Hoffmann, A.A., 1991. Rapid spread of an inherited incompatibility factor in California Drosophila. Nature 353, 440-442.
Turelli, M., Hoffmann, A.A., 1995. Cytoplasmic incompatibility in Drosophila simulans: dynamics and parameter estimates from natural populations. Genetics 140, 1319-1338.

Uyenoyama, M.K., Feldman, M.W., 1980. Theories of kin and group selection: a population genetics perspective. Theoret. Popul. Biol. 17, $380-414$.

van Fraassen, B.C., 1980. The Scientific Image. Oxford University Press, Oxford, UK.

van Fraassen, B.C., 1989. Laws and Symmetry. Oxford University Press, Oxford, UK.

Wade, M.J., 1978. A critical review of the models of group selection. Quart. Rev. Biol. 53, 101-114.

Wade, M.J., 1980. Kin selection: its components. Science 210, 665-667.

Wade, M.J., 1985. Soft selection, hard selection, kin selection, and group selection. Am. Nat. 125, 61-73.

Wade, M.J., 1992. Sewall Wright: gene interaction and the Shifting Balance Theory. In: Futuyma, D., Antonovics, J. (Eds.), Oxford Surveys in Evolutionary Biology, vol. 8. Oxford University Press, Oxford, UK, pp. 35-62.

Wade, M.J., Goodnight, C.J., 1998. Perspective: the theories of Fisher and Wright in the context of metapopulations. When nature does many small experiments. Evolution 52, 1537-1553.

Wade, M.J., Stevens, L., 1994. The effect of population subdivision on the rate of spread of parasite-mediated cytoplasmic incompatibility. J. Theor. Biol. 167, 81-87.

Wade, M.J., Winther, R.G., Agrawal, A.F., Goodnight, C.J., 2001. Alternative definitions of epistasis: dependence and interaction. Trends Ecol. Evol. 16, 498-504.

Werren, J.H., 1997. Biology of Wolbachia. Annu. Rev. Entomol. 42, $587-609$

Werren, J.H., O’Neill, S.L., 1997. The evolution of heritable symbionts. In: O'Neill, S., Hoffmann, A.A., Werren, J.H. (Eds.), Influential Passengers. Inherited Microorganisms and Arthropod Reproduction. Oxford University Press, Oxford, pp. 1-41.

Werren, J.H., Windsor, D.W., Guo, L.R., 1995. Distribution of Wolbachia among neotropical arthropods. Proc. R. Soc. London Ser. B 262, 197-204.

Wimsatt, W.C., 1974. Complexity and organization. PSA 1972 1, 67-86.

Wimsatt, W.C., 1980. Reductionistic research strategies and their biases in the units of selection controversy. In: Nickles, T. (Ed.), Scientific Discoveries: Case Studies. Reidel, Dordrecht, Netherlands, pp. 213-259.

Wimsatt, W.C., 1981. Robustness, reliability and overdetermination. In: Brewer, T., Collins, B. (Eds.), Scientific Inquiry and the Social Sciences. Jossey-Bass, San Francisco.

Wimsatt, W.C., 1987. False models as means to truer theory. In: Nitecki, M.H., Hoffman, A. (Eds.), Neutral Models in Biology. Oxford University Press, Oxford, UK, pp. 23-55.

Wimsatt, W.C., 1994. The ontology of complex systems: levels of organization, perspectives, and causal thickets. Can. J. Philos. 20, 207-274. 